\title{
Toward Coordinated Robust Allocation of Reserve Policies for a Cell-based Power System
}

Hu, Junjie; Heussen, Kai; Claessens, Bert; Sun, Lei; D’Hulst, Reinhilde

Published in:

Proceedings of IEEE Innovative Smart Grid technologies Europe 2016

Link to article, DOI:

10.1109/ISGTEurope.2016.7856307

Publication date:

2016

Document Version

Peer reviewed version

Link back to DTU Orbit

Citation (APA):

Hu, J., Heussen, K., Claessens, B., Sun, L., \& D'Hulst, R. (2016). Toward Coordinated Robust Allocation of Reserve Policies for a Cell-based Power System. In Proceedings of IEEE Innovative Smart Grid technologies Europe 2016 IEEE. https://doi.org/10.1109/ISGTEurope.2016.7856307

\section{General rights}

Copyright and moral rights for the publications made accessible in the public portal are retained by the authors and/or other copyright owners and it is a condition of accessing publications that users recognise and abide by the legal requirements associated with these rights.

- Users may download and print one copy of any publication from the public portal for the purpose of private study or research.

- You may not further distribute the material or use it for any profit-making activity or commercial gain

- You may freely distribute the URL identifying the publication in the public portal 


\title{
Toward Coordinated Robust Allocation of Reserve Policies for a Cell-based Power System
}

\author{
Junjie Hu*, Kai Heussen ${ }^{\dagger}$, Bert Claessens ${ }^{\ddagger}$, Lei Sun ${ }^{\S}$, Reinhilde D’Hulst ${ }^{\Uparrow}$ \\ $*,{ }^{\dagger}$, Center for Electric Power and Energy, Technical University of Denmark, Denmark. \\ $\ddagger$, Restore, Belgium. \\ $\S$, Department of Electrical Engineering, ZheJiang University, China. \\ I, Department of Energy, VITO, Belgium. \\ Email: *junhu@elektro.dtu.dk, ${ }^{\dagger}$ kh@elektro.dtu.dk, ${ }^{\ddagger}$ bert.claessens@ restore.eu, ${ }^{\S}$ eesunlei@ zju.edu.cn, \\ Ireinhilde.dhulst@vito.be,
}

\begin{abstract}
Conventional regulation reserves have fixed participation factors and are thus not well suited to utilize differentiated capabilities of ancillary service providers. This study applies linear decision rules-based (LDR) control policies, which effectively adapt the present participation factor in dependence of the imbalance signal of previous time steps. The LDR-policies are centrally computed using a robust optimization approach which takes into account both the covariances of historic imbalance signals and the operational flexibility of ancillary service providers. The concept is then extended to the cooperation of multiple cells. Two illustrating examples are presented to show the functioning of the proposed LDR method.
\end{abstract}

Index Terms-Linear decision rules, Energy constrained resources, System balancing.

\section{INTRODUCTION}

To reduce greenhouse-gas emissions and fossil-fuel dependency in the power industry, renewable energy resources such as wind and solar are widely advocated and their penetration is increasing in the power system [1], [2]. However, these intermittent energy resources bring challenges to the power system operation due to their intrinsic uncertainty. Certainly in a setting with reducing power system reserves from traditional balancing resources [3]. Furthermore introducing intermittent generation at the medium and low voltage level causes congestion issues. On the other hand, new loads are introduced into the system, such as electric vehicles and heat pumps, which may be managed actively by demand response or other control schemes [4]. In this study, these new loads are generally referred to as distributed energy resources (DERs) and are characterized by energy constrained electric power flexibility [5].

Unlocking the sleeping flexibility of this residential energy constrained flexibility promises a vast resource to mitigate challenges related to the uncertainty of renewable energy generation, both at system and local level. Enabling this flexibility comes at the cost of an increasingly complex control system, characterized by a huge number of state and decision variables. Possible control architectures studied in literature can be described in terms of their control architecture (centralized, distributed, decentralized) [6], their organizational structure (vertical, horizontal), or coordination strategies (cooperative,

978-1-5090-3358-4/16/\$31.00 (C)2016 IEEE non-cooperative, direct, indirect, transactive, etc.) [7]. In particular it is often overlooked that even with conventional hierarchical demand response schemes, the potential value of DER flexibility as a local control resource is hardly addressed. A conventional vertical, direct and centralized, approach of system balancing is therefore not directly transferable, nor appropriate to control a power system with a large number of distributed energy resources. In this paper a control approach is presented merging advantages of the control architectures mentioned above.

The European FP7 project ELECTRA IRP [8] proposes and develops a "Web of Cells" (WoC) architecture for operating the future power system. In this approach, the power systems operation is divided into connected cells, each responsible for their own balancing and voltage control [9], thus establishing a robust, decentralized horizontal decomposition as opposed to the conventional centralized and vertical system operation. The use cases/controllers within the WoC concept are discussed in section II.A.

While the demand side flexibility is becoming resourceful, we observe that, in contrast to conventional controllable generation, a) demand side resources cannot continuously be activated (limited energy flexibility), b) these resources require much shorter planning and reservation periods, as they follow other use patterns, and c) the locality of DER of connection points can be traded off against the benefits of resource sharing and aggregation. With respect to flexibility for balancing the power system, the following issues thus need to be further investigated: $i$ ) when energy constrained units (such as batteries) provide control reserves, their limitation on the energy capacity should be taken into account; ii) reserve allocation should be close to operating time, considering the fluctuating renewable energy resources; and iii) the trade-off between local benefits of flexibility and shared utilization/aggregation needs to be accounted for directly.

In this paper we propose a refinement of the WoC balancing control, addressing $i$ ) and ii) by a robust power system reserve allocation approach based on [10], combining a predictive dispatch with optimal reactive control policies in form of linear decision rules (LDR) [11]. Further, we address iii) by proposing potential coordination strategies across cells. Our 
approach thus exploits both spatial and temporal correlation and smoothing effects of imbalance signals. Compared to existing reserve operation, the operational costs are reduced by a more effective combination of balancing resources as well as reduced reserve volumes.

The rest of the paper is organized as follows: In Section II, the background on the WOC and the linear decision rulebased reserve allocation is described. Section III presents the proposed control architecture where a single cell operation and web of cells concepts are included. In section IV, case studies are reported. Finally, discussion and conclusions are reported in Section V.

\section{BACKGROUND}

As the results present an application of the policy-based reserve concept [10] to the ELECTRA WoC use cases, we outline the fundamentals here.

\section{A. Web of Cells Concept: Fully Decentralized and Distributed Power System Control}

The Web of Cells (WoC) concept [8] is a proposal to reformulate the control architecture of electric power systems to accommodate the challenges of fully distributed generation, reduced inertia, storage integration and flexible demand. The core of $\mathrm{WoC}$ is a reformulation of power system real-time operation into a decentralized control scheme in terms of semiautonomous cells. Cells, as non-overlapping topological subsets of a power system, are associated with a scale-independent operational responsibility to contribute to system operation and stability. This horizontal and decentralized scheme increases the robustness with respect to communication and ensures a 'fallback' operating state in case of significant disruptions. The operating state, including power exchanges and reserve parameters, then, can be continuously optimized by distributed coordination across cells. In contrast with present vertical operating concepts, here operating responsibility is fully delegated to a cell, while in contrast with microgrids, the WoC concept assumes a base case of interconnected operation.

The control concept is defined in terms of four active power control functions and two voltage control functions [9]: a cascaded reactive control from fast inertia response, over frequency containment to balance restoration, eliminates system and cell imbalances, which is then adjusted coordination with neighbouring cells via balance steering. The goal of balance restoration control is to restore control cell balance and by doing so restoring inter-cell power flows to secure values. The aim of balance steering control is to minimise the need for balancing reserves.

The WoC control architecture can be formulated in terms of four control topology levels (CTLs): device level - CTL 0, flexibility unit level (aggregate device flexibility) - CTL1, cell level - CTL2, and WoC level - CTL3. For the subject of this work we focus on CTL1 to CTL3: the objective of balance restoration is defined at CTL2, utilizing resources from CTL1; whereas coordination with neighbouring cells in balance steering corresponds to CTL3 operations utilizing CTL2 entities and possibly CTL1 resources. To address elements at a specific control topology level we define the following notation:

- A flexible unit $i$ corresponds to CTL1 and is an element of the set of all units $i \in \Omega$ in the power system;

- A cell $\alpha$ is composed of a set of units $i \in \phi_{\alpha} \subseteq \Omega$; Note that cells do not share units: $\phi_{\alpha 1} \cap \phi_{\alpha 2}=\emptyset$ for $\alpha_{1} \neq \alpha_{2}$, and each unit belongs to a cell $\bigcup_{\alpha \in \Theta} \phi_{\alpha}=\Omega$;

- The cell is an element $\alpha \in \Theta$ in the set of all cells.

- A cell $\alpha$ can be part of a set of cells $\alpha \in \Phi_{p} \subseteq \Theta$

A further refinement of these sets is provided in Section III-B.

As operational context, the WoC concept assumes predefined operating points for power and thus a market based operating schedule (with e.g. $15 \mathrm{~min}$ time resolution), for all units and estimated exchanges of tie-line power flows. The control architecture accommodates deviations formulated with respect to such a schedule, respecting operating constraints utilizing allocated reserves.

\section{B. Robust Reserve Allocation with Linear Decision Rules}

The power system model and the affine control policies are briefly introduced here, based on the work in [10], more details for the cell operations will be introduced in Section III-A.

1) Node model: The power system consists of a set of nodes connected to the power network. Two types of node models are introduced: inelastic and elastic power injections or extractions. The inelastic part of a node is modeled as $\mathbf{r}_{i}+G_{i} \delta$, where $i$ identifies each participating unit. $\mathbf{r}_{i} \in \mathbb{R}^{T}$ refers to the nominal predicted power injection or extraction. The aggregated random forecast error $\delta$ has the form $\delta \in \mathbb{R}^{T}, \delta=$ $\left[\delta_{0}, \ldots, \delta_{T-1}\right]^{\prime}$, and $G_{i}$ represents the unit's proportional share of $\delta$.

The elastic power part is modeled as $C_{i} \mathbf{X}_{i}$, where $C_{i} \in$ $\mathbb{R}^{T \times n_{i} T}, \mathbf{X}_{i} \in \mathbb{R}^{n_{i} T}$ is the state variable of unit $i, n_{i}$ is the state dimension, which can be written as a function of the input sequence and the current state $x_{0}^{i} \in \mathbb{R}^{n_{i}}$.

$$
\mathbf{X}_{i}=A_{i} x_{0}^{i}+B_{i} \mathbf{u}_{i}
$$

where $\mathbf{u} \in \mathbb{R}^{T}$ is the control input to the elastic power units for balancing the system. The internal state $x_{i}$ is governed by linear time-invariant dynamics modeled by $A_{i} \in \mathbb{R}^{n_{i} \times n_{i}}$ and $B_{i} \in \mathbb{R}^{n_{i}}$.

2) network model: In the network model part, a system balance equality constraint is imposed for the inelastic power flow and elastic power flows, thermal lines constraints can also be included.

3) Affine control policies: To formulate the optimal reserve allocation minimization problem, a quadratic cost function including the control variable $\mathbf{u}$ and state variable $\mathbf{x}$ is used. To make the optimization problem tractable, policies of affine form are applied which leads to the following equation $\mathbf{u}_{\mathbf{i}}=\mathbf{e}_{\mathbf{i}}+D_{i} \delta$ where $\mathbf{e}_{\mathbf{i}} \in \mathbb{R}^{T}$ is the nominal schedule for elastic unit $i$, and $D_{i} \in \mathbb{R}^{T \times T}$ is the dynamic responding part to the prediction errors. $D_{i}$ takes the lower-triangular form. 


\section{LiNEAR DECISION RULES FOR ALLOCATING RESERVES IN A CELL-BASED POWER SYSTEMS}

In this section, we firstly introduce the robust optimal reserve allocation problem with linear decision rules at the level of a single cell (CTL2). In a second step, we propose an extension of this application to include multiple cells, thus defining inter-cell cooperation models (CTL3).

\section{A. Single Cell Operation}

A cell imbalance is computed based on the cell boundary integral (i.e. the sum of tie-line flows), as compared to scheduled flows and corrected for the cell's power-frequency characteristic. The imbalance of a single cell $\alpha$ is the sum of a forecasted deviation and further real-time fluctuations:

$$
\boldsymbol{\Delta}_{\alpha}=\sum_{i \in \phi_{\alpha}}\left(\mathbf{r}_{i}+G_{i} \delta_{\alpha}\right)=\mathbf{r}_{\alpha}+\delta_{\alpha} \quad \forall \alpha \in \Theta
$$

Balancing power provided by a cell is based:

$$
\mathbf{u}_{\alpha}=\sum_{i \in \phi_{\alpha}} \mathbf{u}_{i}=\sum_{i} \mathbf{e}_{i}+D_{i} \delta_{\alpha} \quad \forall \alpha \in \Theta
$$

1) Cell-based reserve allocation model: In this cell-based power system, four types of units are considered and these four types of units are represented in four sets $\phi_{\alpha}^{\mathrm{RE}}, \phi_{\alpha}^{\mathrm{L}}, \phi_{\alpha}^{\mathrm{G}}$ and $\phi_{\alpha}^{\mathrm{DER}}$. In detail,

1) Renewable generation, which brings the uncertainty to the system. Wind power is modeled as $W_{i}+G_{i} \delta_{\alpha}$, where $W_{i}$ denotes the nominal prediction and $G_{i} \delta_{\alpha}$ represents wind proportion of the forecast error whose value is to be discovered in the future.

2) General load, which can be modeled as $P_{i}^{\text {load }}+G_{i} \delta_{\alpha}$ the physical meaning of these variables is similar to the one in the wind generation.

3) Thermal generation that matches the needs of the general loads as well as responding to the uncertainty introduced by the wind generation. Thermal generation is modeled as $P_{i}^{g}\left(\delta_{\alpha}\right) \in \mathbb{R}^{T}$.

4) Distributed energy resources with flexibilities like electric vehicles, batteries etc. These are considered as energy constrained resources. A distributed energy resource is modeled as $P_{i}^{\text {der }}\left(\delta_{\alpha}\right) \in \mathbb{R}^{T}$.

For a typical reserve allocation problem, the basics are well explained in [12] such as the system balance constraint, the thermal generation power constraints etc., thus this will not further be explained here.

Specifically, regarding unit type 4), it is assumed that an aggregator a portfolio of flexible energy-constrained resources, represented here as a single DER unit. As a portfolio, these energy constrained resources can shift their power over time, but are constrained by a maximum (and minimum) energy consumption. Examples of such resources are electric vehicles, batteries, etc. The individual energy constraints of such a resource are specified by two bounds, $E_{\text {min }}$ and $E_{\text {max }} \in \mathbb{R}^{T}$, expressing the maximum allowed and minimally required energy consumption of the resource for each timestep within the applied time horizon $T$. The aggregator portfolio
(DER unit) is also bounded by power constraints $\left(P_{i, \text { min }}^{\text {der }}\right.$ and $P_{i, \text { max }}^{\text {der }} \in \mathbb{R}^{T}$ ) indicating the maximum and minimum power the portfolio can consume/produce at each instant during time $T: P_{i, \min }^{\text {der }} \leq P_{i}^{\text {der }}\left(\delta_{\alpha}\right) \leq P_{i, \max }^{\text {der }}$. The combination of energy and power constraints are an expression of the flexibilityboundaries of the unit:

$$
E_{i, \min } \leq E_{0}+M P_{i}^{d e r}\left(\delta_{\alpha}\right) \Delta t \leq E_{i, \max }
$$

where $M \in \mathbb{R}^{T \times T}$ with $M_{i, j}=1$ for $i \geq j$ and $M_{i, j}=0$ for $i<j$.

Overall, the reserve-based optimization problem is formulated as follows:

$$
\begin{aligned}
\min & \mathbb{E}_{\delta \alpha}\left\{\sum_{i \in \phi_{\alpha}^{\mathrm{G}}}\left(\alpha_{i}^{g} P_{i}^{g}\left(\delta_{\alpha}\right)^{T} P_{i}^{g}\left(\delta_{\alpha}\right)+B_{i}^{g} P_{i}^{g}\left(\delta_{\alpha}\right)+\overline{\mathbf{I}} C_{i}\right)+\right. \\
& \left.\sum_{i \in \phi_{\alpha}^{\mathrm{DER}}}\left(\alpha_{i}^{d e r} P_{i}^{d e r}\left(\delta_{\alpha}\right)^{T} P_{i}^{d e r}\left(\delta_{\alpha}\right)+B_{i}^{d e r} P_{i}^{d e r}\left(\delta_{\alpha}\right)+\overline{\mathbf{I}} C_{i}\right)\right\}
\end{aligned}
$$

subject to system constraints, line constraints, and individual unit's operational constraints, where $a_{i}^{g, d e r}, B_{i}^{g, d e r}, C_{i}$ are stacked vector of quadratic, linear coefficients and constant part of the generation/DER cost function, $\overline{\mathbf{I}}$ is a vector of all ones. Until this stage, the whole optimization problem is intractable due to the wide variety of candidate functions $P_{i}^{g}\left(\delta_{\alpha}\right), P_{i}^{\text {der }}\left(\delta_{\alpha}\right)$.

2) Robust allocation with Linear Decision Rules: We restrict ourselves to the case where recourse decision variables $P^{g}\left(\delta_{\alpha}\right), P_{i}^{\text {der }}\left(\delta_{\alpha}\right)$ are affine functions of uncertainty $\delta_{\alpha}$ [13]. This implies the power schedule of thermal generation and distributed energy resources comply with the following relationships:

$$
\begin{gathered}
P_{i}^{g}\left(\delta_{\alpha}\right)=e_{i}^{g}+D_{i}^{g} \delta_{\alpha}, \forall i \in \phi_{\alpha}^{\mathrm{G}} . \\
P_{i}^{d e r}\left(\delta_{\alpha}\right)=e_{i}^{d e r}+D_{i}^{d e r} \delta_{\alpha}, \forall i \in \phi_{\alpha}^{\mathrm{DER}} .
\end{gathered}
$$

where the upper diagonal part of matrix $D$ is zero.

In the remainder of this section, we introduce reformulations of the objective function as an example for applying this affine functions.

Substituting (6) and (7) into the objective function (5), one gets

$$
\begin{aligned}
\min \mathbb{E}_{\delta \alpha}\{ & \sum_{i \in \phi_{\alpha}^{\mathrm{G}}}\left(\alpha_{i}^{g}\left(e_{i}^{g}+D_{i}^{g} \delta_{\alpha}\right)^{2}+B_{i}^{g}\left(e_{i}^{g}+D_{i}^{g} \delta_{\alpha}\right)\right. \\
& \left.+\overline{\mathbf{I}} C_{i}\right)+\sum_{i \in \phi_{\alpha}^{\mathrm{DER}}}\left(\alpha_{i}^{d e r}\left(e_{i}^{d e r}+D_{i}^{d e r} \delta_{\alpha}\right)^{2}\right. \\
& \left.\left.+B_{i}^{d e r}\left(e_{i}^{d e r}+D_{i}^{d e r} \delta_{\alpha}\right)+\overline{\mathbf{I}} C_{i}\right)\right\}
\end{aligned}
$$

With $\mathbb{E}\left[\delta_{\alpha}\right]=0$ and the known $\mathbb{E}\left[\delta_{\alpha} \delta_{\alpha}^{\prime}\right]$, the objective function (8) can be written as 


$$
\begin{aligned}
& \min \sum_{i \in \phi_{\alpha}^{\mathrm{G}}}\left(\alpha_{i}^{g}\left(e_{i}^{g T} e_{i}^{g}+\left\langle D_{i}^{g T} D_{i}^{g} \mathbb{E}\left[\delta_{\alpha} \delta_{\alpha}^{\prime}\right]\right\rangle\right)+B_{i}^{g} e_{i}^{g}+\right. \\
&\left.\overline{\mathbf{I}} C_{i}\right)+\sum_{i \in \phi_{\alpha}^{\mathrm{DER}}}\left(\alpha _ { i } ^ { d e r } \left(e_{i}^{d e r T} e_{i}^{d e r}+\right.\right. \\
&\left.\left.\left\langle D_{i}^{d e r T} D_{i}^{d e r} \mathbb{E}\left[\delta_{\alpha} \delta_{\alpha}^{\prime}\right]\right\rangle\right)+B_{i}^{d e r} e_{i}^{d e r}+\overline{\mathbf{I}} C_{i}\right)
\end{aligned}
$$

For other equality and inequality constraints reformulation, the approach is similar to [13] and thus the detailed formulation is not presented here.

\section{B. Co-operation of Multiple Cells}

Whereas power system operation today assumes automatic support for low-voltage subsystems to be provided from higher voltage levels, the WoC, in a first step, removes this assumption and replaces it with a new strong responsibility for cells: to maintain balance with respect to exchange schedules and other operational constraints. However, as discussed above, this WoC framework may also be employed to define cooperation strategies across cells (i.e. at CTL3). Together separation of concerns enables overall more secure operation and a modularized approach to handle the increased number decision variables. In this section we will demonstrate how cooperation amongst cells by (mutual) assistance with imbalances can be defined, and explore a few simple cooperation strategies. We define the cooperation in two steps: first a cooperation topology is formulated, and second, the cooperation strategy on this topology is defined.

1) Coordination topology: For cell operators, a cooperative behaviour is likely, as operators share goals and are noncompeting. In balancing control, cooperation corresponds to active power being transferred from one cell to one or several others to reduce other's or a shared imbalance. A coordination topology in a Web-of-Cells can then be thought of as unidirectional (directed graph) or as non-directional (undirected graph). The general (directional) case is formalized via adjacency sets as follows:

- for each cell $\alpha$ the set $\mathcal{N}_{\alpha} \subseteq \Theta$ refers to the cells supported by it, i.e. by responding in some way to the imbalances of a cell $\beta \in \mathcal{N}_{\alpha}$.

- further, each cell $\alpha$ there is a set $\mathcal{M}_{\alpha} \subseteq \Theta$ of cells it is supported by.

Obvious special cases are:

Case 1: No cooperation between cells: $\left\|\mathcal{M}_{\alpha}\right\|=\left\|\mathcal{N}_{\alpha}\right\|=1$ for all $\alpha \in \Theta$, with $\{\alpha\}=\mathcal{M}_{\alpha}$ and $\{\alpha\}=\mathcal{N}_{\alpha}$. This case corresponds to a replication of [previous section] in all cells. It is the 'fully decentralized' solution. While computationally feasible, this case is suboptimal, considering the potential for imbalance netting and resource optimization across cells.

Case 2: Full cooperation between all cells: $\mathcal{M}_{\alpha}=\mathcal{M}_{\beta}=\Theta$ and thus $\mathcal{N}_{\alpha}=\mathcal{N}_{\beta}=\Theta$ for all $\alpha, \beta \in \Theta$. This case may be an extreme case if the set of cells is large, and may thus not be tractable as a single optimization problem.
In between these extreme cases there is a range of possible directional or non-directional cooperation topologies. Such cooperation would, as one criterion, be related to the electrical topology of the power system - but also other criteria should be considered, such as e.g. complementarity of balancing resources or (anti-)correlation of respective imbalance signals.

In the following we shall focus on one non-directional cooperation topology referred to as pool of cells: A cell $\alpha$ can be part of a pool of cooperating cells $\alpha \in \Phi_{p} \subseteq \Theta$; this term implies a mutual support role such that a mix of both special cases above applies for all cells in $\Phi_{p}$ : $\mathcal{M}_{\alpha}=\mathcal{M}_{\beta}=\Phi_{p}, \mathcal{N}_{\alpha}=\mathcal{N}_{\beta}=\Phi_{p}$ for all $\alpha, \beta \in \Phi_{p}$, and thus $\left\|\mathcal{M}_{\alpha}\right\|=\left\|\mathcal{N}_{\alpha}\right\|=\left\|\Phi_{p}\right\|$.

In other words, within a pool of cells the participating cells pool both imbalances and balancing resources together.

Another situation with high practical relevance is the case in which the asymmetric pooling is likely: e.g. for densely populated (urban) distribution grids, it is unlikely that the generation and balancing resources are sufficient to help out another cell, whereas, propagating todays operating principles, it will likely require external balancing resources. Such a case corresponds to a pool of cells helping a single cell: $\mathcal{M}_{\alpha}=\Phi_{p}$, and the cell only helping itself $\left\|\mathcal{N}_{\alpha}\right\|=1$ with $\{\alpha\}=\mathcal{N}_{\alpha}$. Such cases can also be formulated as a cascade with several pools, or in the sense of a single 'higher-level' cell helping several 'appended' cells: : $\mathcal{N}_{\alpha}=\Phi_{p}$, and the cell's own imbalance not being a concern $\left\|\mathcal{M}_{\alpha}\right\|=0$.

2) Coordination Strategy: With a given cooperation topology, we can introduce the coordination strategy, which defines how the cooperation is realized. Two kinds of coordination strategies are considered in this study: a) imbalance-pooling cooperation, in which the cells of a pool $\Phi_{p}$ form a joint imbalance signal $\left(\delta_{p}=\sum_{\alpha \in \Phi_{p}} \delta_{\alpha}\right)$ that is balanced jointly by also poolung the balancing resources (cf. Scenario 2 of Section IV. A); b) balancing support, in which support is provided asymmetrically (corresponding to the last case discussed above); this case is studied in Scenario 3 of Section IV.A.

\section{CAse Studies}

In this section, we firstly present the case study for a threecell system, investigating different coordination strategies. Then, the single cell case study is presented where two types of units are used to balance out the cell imbalance, and features of energy constrained resources are described.

\section{A. Multiple (2-3) cells with grid constraints and energy con- strained units}

Fig. 1 shows an illustrating example of a three cells system, in which the three cells are connected by two tie-lines. Three scenarios in terms of coordination strategies are envisioned in this case study: 1) a fully decentralized solution where each cell has individual imbalance signals and has enough resources to address the imbalances within the cell; 2) cell 1 and cell 2 have a joint imbalance signal and thus the imbalance will be addressed by the joint resources of cell 1 and cell 2, cell 0 has 


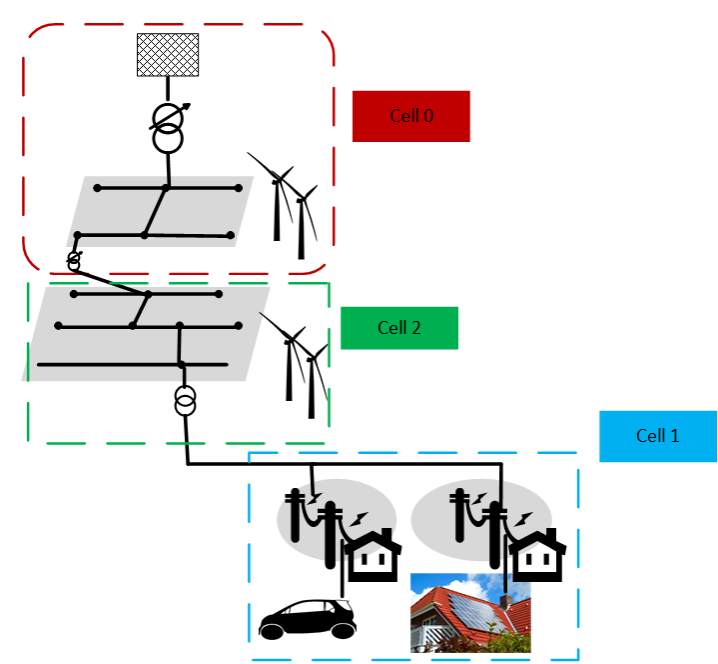

Fig. 1: An illustrating example of three cells system
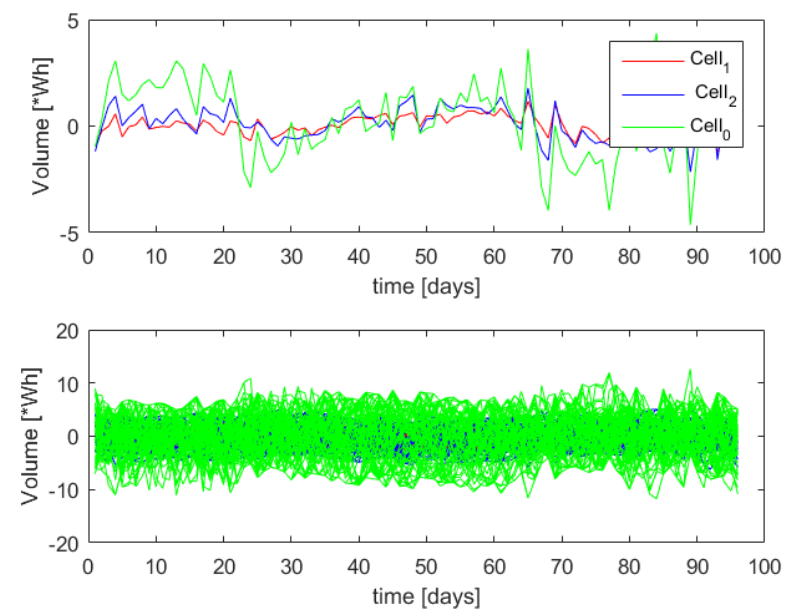

Fig. 2: An illustrating example of three cells system

its imbalance signal and its own balancing resources; 3) this scenario is a variant of scenario 1 but in this scenario cell 1 balancing resources can be used to address the imbalances in both cell 1 and cell 2 .

To find a reasonable imbalance signal for the system, the historical data of 15-mins based Belgian system imbalance (available on the website of the Belgian TSO Elia [14]) is scaled down and used for this study. The half year timeseries imbalance signals are divided into three segments. When adapting the signals to the three cells, the scaling down factor is increased by a product of 2 when it comes to cell 0 , cell 2 , and cell 1. Afterwards, the mean of the scaled value in each segment is used as the nominal predictions for three cells, which is shown in the Fig. 2. The prediction error for each cell was defined as the deduction of the scaled value and the mean, which is also shown in the Fig. 2. Note that for scenario 2 , the imbalance signals of cell 1 and cell 2 are combined into one imbalance signal.
The prediction error covariance of the three cells is calculated and an operation period of three hours is selected in the study. In term of the coefficients of the cost functions for the balancing resources within each cell (note an aggregated balancing resource/battery is assumed for each cell in this three-scenario comparison study), the quadratic and linear coefficients of the resource for cell 1 , cell 2 and cell 0 are $(0.1,0.1),(1,3),(0.1,5)$, respectively. Here, we envision that cell 1 will have balancing resources consisting of electric vehicles, batteries, heat pumps etc. Cell 2 balancing resources could come from industrial demand response and a dedicated battery, and the resources of cell 0 mainly are conventional thermal generations. By having the power constraints of the balancing resources and the compact set of the prediction error constraints, the balancing cost for the three scenarios are calculated as 120.67 for scenario 1, 22.29 for scenario 2, and -297.08 for scenario 3. These results indicate that scenario 2 and 3 show a clear advantage over scenario 1 in term of having a lower cost because of the joint imbalance signals as well as the cooperation strategy. Scenario 3 is interesting because of the negative value of the cost. This is because when addressing the imbalances in cell 1 and cell 2 , especially in cell 1 , the optimization tries to charge battery of cell 1 a lot and meanwhile discharge the battery of cell 0 significantly with the purpose of reducing the balancing cost, considering the huge cost difference between cell 1 and cell 0 . Note that energy constraints, tie-line flow and other practical constraints are not considered here, this may change the value of the reserve cost but the reasoning remains.

\section{B. Single Cell with Energy Constraint units}

In this single cell operation, two types of units/batteries at control topology level 1 (CTL1) are included for demonstrating the LDR policy as well as the features of the energy constrained resources. These two types of units are unit 1, and unit 2 which is energy constrained. It is assumed that reserve cost of unit 2 is cheaper than unit 1 . The quadratic and linear coefficients for two units are $(0.3,0.5),(0.1,0.5)$.

The brighter yellow diagonal of lower part of Fig. 3 indicates that unit 1 contribute more than unit 2 to the cell imbalances, even though the cost of unit 1 is expensive. This is because, as illustrated in Fig. 4, the energy capacity of unit 2 has been nearly reached at the end of the planned horizon. In the figure, blue coarse curve means the minimum energy capacity of unit 2 , red coarse curve is the maximum energy capacity of unit 2 , red thin curve is the response curve to the worst positive imbalance signal while the green curve denotes the response to the worst negative imbalance signal. The black curves are realizations of 10 typical three hours (15 minsbased) curves randomly chosen from the half year used in generating the covariances. The energy dynamics of two units show the robustness of the approach.

\section{CONClusion}

We presented an application of a recent method for calculation of robust policy based reserves to the ELECTRA Web-of- 

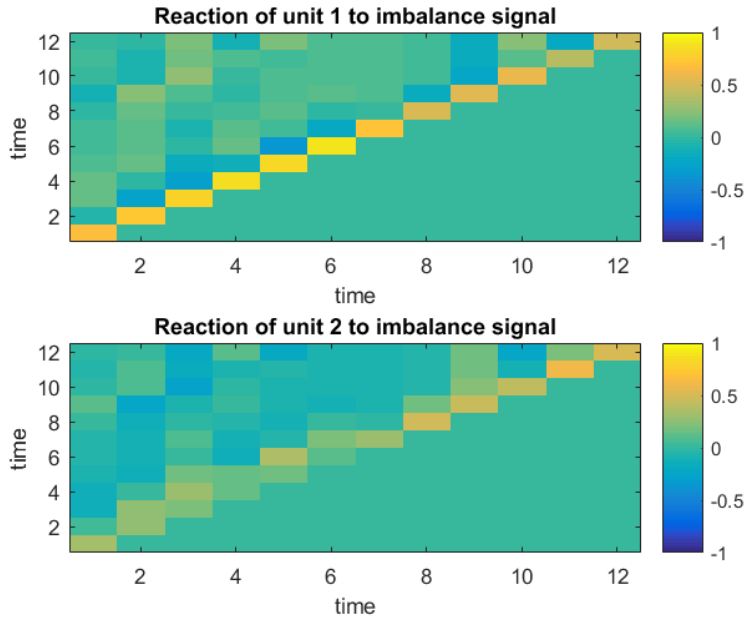

Fig. 3: Reactions of unit 1 to the imbalance signals.
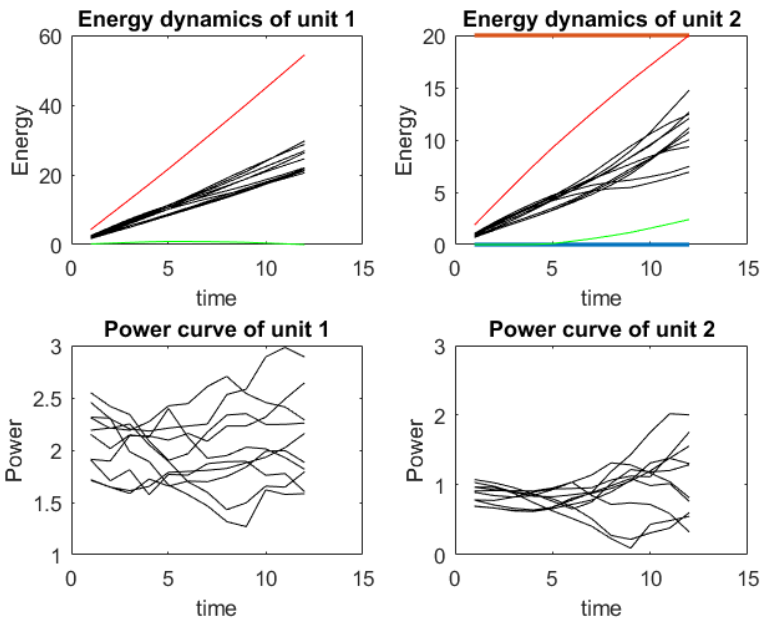

Fig. 4: Energy and power curves of two units.

Cells control architecture and explored basic schemes of intercell coordination. By exemplary comparison of the basic decentralized scheme with two alternative inter-cell coordination schemes the potential resource savings of cooperation have been revealed. Further, it has been demonstrated that control policies based on Linear Decision Rules are well suited for integration of energy-constrained DER in reactive balancing control, and the robust allocation method is applicable to realistic imbalance signals. Future work should develop additional inter-cell coordination schemes and explore the potential multi-level aggregation of control resources in a policy-based control framework. In addition, when adapting the method into real cases, the cost function that represents the balancing service provision should also be investigated.

\section{ACKNOWLEDGMENT}

The research leading to these results has received funding from the European Union Seventh Framework Programme
[FP7/2007-2013] under grant agreement n'609687, and includes support from the ELECTRA REX researcher exchange programme. Junjie Hu also thanks Chris Caerts (VITO) and Mattia Marinelli (DTU) for the support on his research exchange at VITO.

\section{REFERENCES}

[1] I. E. Agency, "Renewable energy medium-term market report 2015. Market Analysis and Forecasts to 2020," 2015.

[2] I. Pineda and J. Wilkes, "Wind in Power: 2014, European Statistics," Technical Report, no. February, pp. 1-12, 2015.

[3] P. De Martini, K. Mani Chandy, and N. Fromer, "Grid 2020 Towards a Policy of Renewable and Distributed Energy Resources," Tech. Rep. September, The Resnick Sustainability Institute at Caltech, California, 2012.

[4] J. Hu, S. You, M. Lind, and J. Østergaard, "Coordinated charging of electric vehicles for congestion prevention in the distribution grid," IEEE Transactions on Smart Grid, vol. 5, pp. 703-711, March 2014.

[5] T. Borsche, A. Ulbig, and G. Andersson, "A new frequency control reserve framework based on energy-constrained units," in Power Systems Computation Conference (PSCC), 2014, pp. 1-7, IEEE, 2014.

[6] D. S. Callaway and I. A. Hiskens, "Achieving controllability of electric loads," Proceedings of the IEEE, vol. 99, pp. 184-199, Jan 2011.

[7] J. Hu, H. Morais, T. Sousa, and M. Lind, "Electric vehicle fleet management in smart grids: A review of services, optimization and control aspects," Renewable and Sustainable Energy Reviews, vol. 56, pp. 1207 - 1226, 2016.

[8] ELECTRA IRP, "European liaison on electricity committed towards research activity integrated research programme.," [Online]. Available: http://www.electrairp.eu/.

[9] R. D'hulst, J. Verbeeck, C. Caerts, M. H. Syed, A. Zaher, and G. M. Burt, "Frequency restoration reserves: Provision and activation using a multi-agent demand control system," in Smart Electric Distribution Systems and Technologies (EDST), 2015 International Symposium on, pp. 601-605, Sept 2015.

[10] J. Warrington, P. Goulart, S. Mariéthoz, and M. Morari, "Policy-based reserves for power systems," IEEE Transactions on Power Systems, vol. 28, pp. 4427-4437, Nov 2013.

[11] D. Bertsimas, D. B. Brown, and C. Caramanis, "Theory and applications of robust optimization," SIAM review, vol. 53, no. 3, pp. 464-501, 2011.

[12] D. Bertsimas, E. Litvinov, X. A. Sun, J. Zhao, and T. Zheng, "Adaptive robust optimization for the security constrained unit commitment problem," Power Systems, IEEE Transactions on, vol. 28, no. 1, pp. 52-63, 2013.

[13] M. Zugno, J. M. Morales, and H. Madsen, "Robust management of combined heat and power systems via linear decision rules," in Energy Conference (ENERGYCON), 2014 IEEE International, pp. 479-486, IEEE, 2014.

[14] Elia [Online], www.elia.be. 